\title{
Angiogenesis in gastric cancer: Importance of the thymidine phosphorylase expression of cancer cells as an angiogenic factor
}

\author{
HYE SEUNG HAN ${ }^{1,2}$ and TAE SOOK HWANG ${ }^{1}$ \\ ${ }^{1}$ Department of Pathology, Konkuk University Hospital, Konkuk University School of Medicine, Seoul 143-729; \\ ${ }^{2}$ Institute of Biomedical Science and Technology, Konkuk University, Seoul 143-701, Korea
}

Received August 7, 2006; Accepted October 30, 2006

\begin{abstract}
Thymidine phophorylase (TP) has been reported to stimulate angiogenesis in a variety of human malignancies. We investigated TP expression and its association with angiogenesis in 73 cases of resected gastric cancer. In addition, we compared the expression of the other angiogenesis related factors (VEGF, eNOS and p53) with that of TP with respect to angiogenesis. TP expression was not detected in most of the non-tumoral glandular epithelial cells except for 5 cases. TP expression of the cancer cells and the stroma was assessed separately. The stromal TP expression was not associated with the TP expression of the cancer cells. The mean percent of TP reactive cancer cells was 18.36 \pm 2.61 (median, 10.00; range, 0-90) and cases showing a percentage higher than the mean were considered as bearing high reactivity. The mean microvessel score assessed was 90.44 \pm 3.69 (median, 86; range, 31-174). The TP expression of cancer cells was strongly associated with microvessel density $(\mathrm{p}=0.030)$, but the stromal TP expression was not. The microvessel density of the tumor showed strong correlation with VEGF expression $(\mathrm{p}<0.001)$, but a marginally significant association with eNOS $(\mathrm{p}=0.055)$. On the contrary, there was no association with p53 expression and microvessel density of the tumor. No significant correlation was detected between lymph node metastasis and tumoral or stromal TP expression or VEGF/TP coexpression. In gastric
\end{abstract}

Correspondence to: Dr Tae Sook Hwang, Department of Pathology Konkuk University Hospital, 4-12 Hwayang-dong, Gwangjin-gu, Seoul 143-729, Korea

E-mail: tshwang@kuh.ac.kr

Abbreviations: TP, thymidine phosphorylase; VEGF, vascular endothelial growth factor; eNOS, endothelial nitric oxide synthase; IHC, immunohistochemistry

Key words: gastric cancer, immunohistochemistry, thymidine phosphorylase, vascular endothelial growth factor, endothelial nitric oxide synthase, p53, angiogenesis cancer, TP expression of the cancer cells, not stromal cells may play an important role in tumor growth by microvessel formation.

\section{Introduction}

Thymidine phosphorylase (TP) has been reported to be produced from a gene known as platelet-derived endothelial cell growth factor (PD-ECGF) (1), which was first isolated from platelets (2) and have a mitogenic and angiogenic potential $(2,3)$. TP may promote angiogenesis in tumors by reducing the intracellular level of thymidine, an inhibitor to endothelial proliferation via the salvage pathway (4). TP expression was found to inhibit apoptosis of cells exposed to hypoxia (5). TP expression provides an advantage for tumor growth in human gastric carcinomas, not only by forming a favorable, highly vascular intratumoral microenvironment, but also by allowing cells to escape the apoptotic mechanism. The expression of TP has been analyzed in a variety of human cancers and has been reported to correlate with high microvessel density and poor prognosis (6-9). Most of the previous reports regarding TP expression in gastric cancers were mainly focused on cancer cells (9-13). However, there were a few reports which analysed TP in cancer cells and stroma separately (10).

The angiogenic ability of the tumors is closely related to their invasive and metastatic potential. Although the role of eNOS in angiogenesis and progression of gastric cancer remains unclear, several studies reported a concentrated localization of eNOS in the endothelial cells of normal gastric mucosa and tumor tissue $(14,15)$. However, a direct association of VEGF and/or of TP with increased intratumoral angiogenesis and poor outcome is rather well established in several studies on early gastric cancer (16-19) and mutations of the $\mathrm{p} 53$ protein have also been suggested to contribute to the angiogenic process through suppression of a strong inhibitor of angiogenesis, namely thrombospondine-1 (20). TP expression in advanced gastric carcinoma has been barely studied.

We investigated TP, VEGF, eNOS, p53 expression and their association with angiogenesis in 73 cases of resected gastric cancer by immmunohistochemical study. We evaluated the TP expression of cancer cells and stroma independently. In 
addition, we evaluated the relationship between TP expression and regional lymph node metastasis.

\section{Materials and methods}

Patients. Seventy-three appropriately preserved samples from 73 patients (50 males and 23 females: mean age, 58.21 \pm 1.41 years) who underwent radical gastrectomy for advanced gastric cancer from September 2000 to July 2001 at the Department of General Surgery, Inha University Hospital, were included in this study. Fundamental data of the patients such as age and sex were retrieved from the data file, and regional lymph node involvement was evaluated by pathologists. The status of the metastatic lymph node was classified according to the Union International Contra la Cancrum (UICC) tumor node metastasis (TNM) staging categories (21). This study was approved by the Ethics Committee of Inha University Hospital.

Tissue preparation. Immediately after resection, the specimens were transported to the Department of Pathology, sampled from the tumor and adjacent normal mucosa, and fixed in absolute methanol (Hayamn, UK) for $24 \mathrm{~h}$. After fixation the tissues were processed in an automatic tissue processor for $12 \mathrm{~h}$.

Immunohistochemistry (IHC). Monoclonal thymidine phosphorylase (p-GF.44C; Oncogene, San Diego, CA, USA), monoclonal CD31 (clone JC/70A ; Dako, Glostrup, Denmark), monoclonal VEGF (clone G153-694; Pharmingen, San Diego), monoclonal eNOS (clone 3; Transduction Lab, Lexington, KY, USA) and monoclonal p53 (clone DO-7; Novocastra, Newcastle, UK) were used for IHC. IHC was performed by a Microprobe Immunostaining System (Foster, CA, USA, Biomeda). Slides were deparaffinized in xylene rehydrated through graded ethanol (100\%-100\%-95\%-95\%), and adequately rinsed with distilled water. After washing, slides were paired together to form a capillary gap, put in the holder, and immersed in $0.05 \mathrm{M}$ Tris buffer containing $0.05 \%$ Tween- 20 . Endogenous peroxidase was quenched by immersion in $3 \%$ hydrogen peroxide for $5 \mathrm{~min}$ at $45^{\circ} \mathrm{C}$, and washed 4 times with $0.05 \mathrm{M}$ Tris buffer containing $0.05 \%$ Tween-20. For all reactions, an LSAB kit (Dako, Carpinteria, CA, USA) was used according to the manufacturer's instructions. After blocking with serum, primary antibody was immediately applied for $1 \mathrm{~h}$ at $45^{\circ} \mathrm{C}$ (TP 1:2000, CD31 1:100, VEGF 1:500, eNOS 1:500, p53 1:100) and biotinylated anti-rabbit and anti-mouse immunoglobulin was used as a secondary antibody. After conjugation with peroxide-conjugated streptavidin for $5 \mathrm{~min}$ at $45^{\circ} \mathrm{C}$, the color was developed by $0.05 \% 3,3$-diaminobenzidine (Sigma, St. Louis, USA). For all assays, positive controls with known tissue samples, and negative controls replacing mouse immunoglobulin for the primary antibody were used.

Immunohistochemical analysis. For the microvessel counting, the areas of the highest vascularization were chosen at low power (x100) and microvessel counting followed on three chosen $x 200$ fields $\left(0.785 \mathrm{~mm}^{2}\right.$ per field $)$ of the highest density. The microvessel score was the mean of the vessel counts obtained in these three fields. Vessels with a clearly defined lumen or well-defined linear vessel shape but not single endothelial cells were taken into account for microvessel counting. The TP expression of the cancer cells and the stroma was assessed separately. We examined at least 200 carcinoma cells to determine whether the cells were positive for TP at high power (x400) after screening for the areas with the highest intensity of staining at lower power $(\mathrm{x} 100)$. The mean value of positive cells was used as a cut-off point to distinguish between cases with low and high reactivity. The expression of TP by stromal fibroblasts and infiltrating cells was also assessed. Intense stromal TP expression in more than $50 \%$ of optical (x200) fields examined was used as a criterion to define high versus low TP stroma reactivity. The degree of positivity of VEGF, eNOS and p53 was graded by a semiquantitative approach. The proportion of positively stained tumor cells to the total number of tumor cells was graded as follows: negative, $<10 \%$; low expression, $10-50 \%$; high expression, $>50 \%$ positively stained tumor cells. Concerning p53 expression, the cases with $>10 \%$ of positive nuclear staining of cancer cells were considered as positive. Two pathologists reviewed the results independently. During the evaluation, both of the investigators were unaware of the clinical outcome of the patients. Discrepancies were resolved on the conference microscope.

Statistical analysis. We investigated TP expression and its association with angiogenesis and lymph node metastasis in 73 cases of resected gastric cancer by the SPSS 10.0 statistical software package (SPSS Inc., Chicago, IL, USA.). Student's t-test was performed to analyze the association between microvessel density and TP expression of cancer cells, TP expression of stroma, VEGF, eNOS and p53. Trend test was performed to analyze the relationship between TP expression and regional lymph node involvement. The accepted level of statistical significance was $\mathrm{p}<0.05$.

\section{Results}

Result of immunostaining. TP expression was not detected in most of the non-tumoral glandular epithelial cells except for 5 cases (Fig. 1A). Immunoreactivity for TP was noted in the nuclei and the cytoplasm of cancer cells and stromal cells, and stromal TP expression was not associated with the TP expression of the cancer cells (Fig. 1B and C). The mean percent of TP reactive cancer cells was $18.36 \pm 2.61$ (median, 10.00; range, 0-90) and cases showing a percentage higher than the mean were considered as bearing high reactivity. TP expression in the cancer cells was low in $47(64.4 \%)$ and high in $26(35.6 \%)$ of 73 cases. TP expression in the stroma was low in $42(57.5 \%)$ and high in $31(42.5 \%)$ of 73 cases. The mean microvessel score assessed was 90.44 \pm 3.69 (median, 86; range, 31-174). VEGF expression was seen in the cytoplasm of the cancer cells and the endothelial cells (Fig. 2A). VEGF expression was low in $22(30.1 \%)$ and high in $51(69.9 \%)$ of 73 cases. Generally the eNOS expression of the endothelial cells was stronger than that of cancer cells (Fig. 2B). eNOS expression was also noted in the cytoplasm of endothelial cells of normal mucosa. eNOS expression was low in 47 (64.4\%) and high in $26(35.6 \%)$ of 73 cases. p53 expression was seen in the nuclei of the cancer cells (Fig. 2C). Thirty-one (42.5\%) of 73 cases were negative and $42(57.5 \%)$ were positive. 

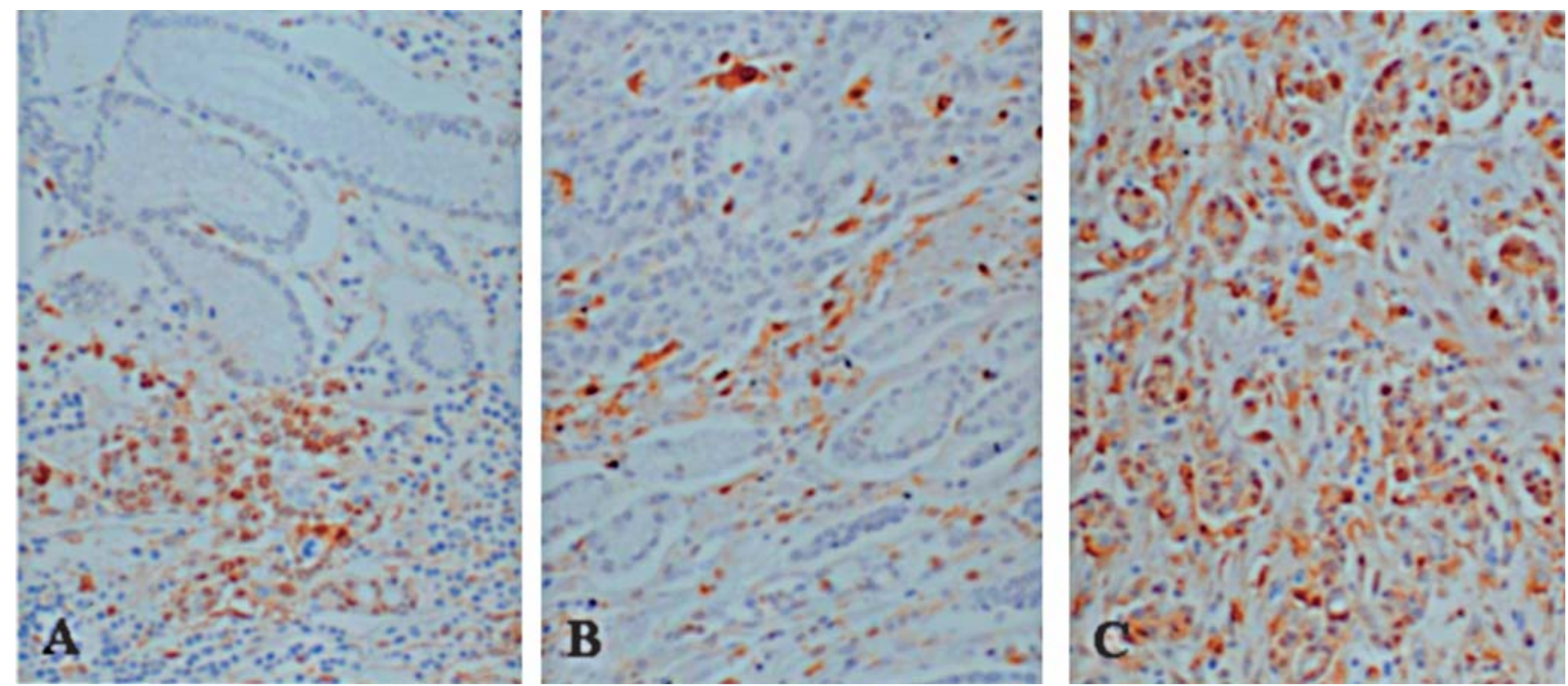

Figure 1. Strong thymidine phosphorylase expression in cancer cells and negative expression in normal glands. A few stromal cells are also stained (A). Negative thymidine phosphorylase expression in both normal glands and cancer cells. Stromal cells scattered within the tumor cells are intensely stained (B). Strong thymidine phosphorylase expression in both cancer cells and stromal cells (C).
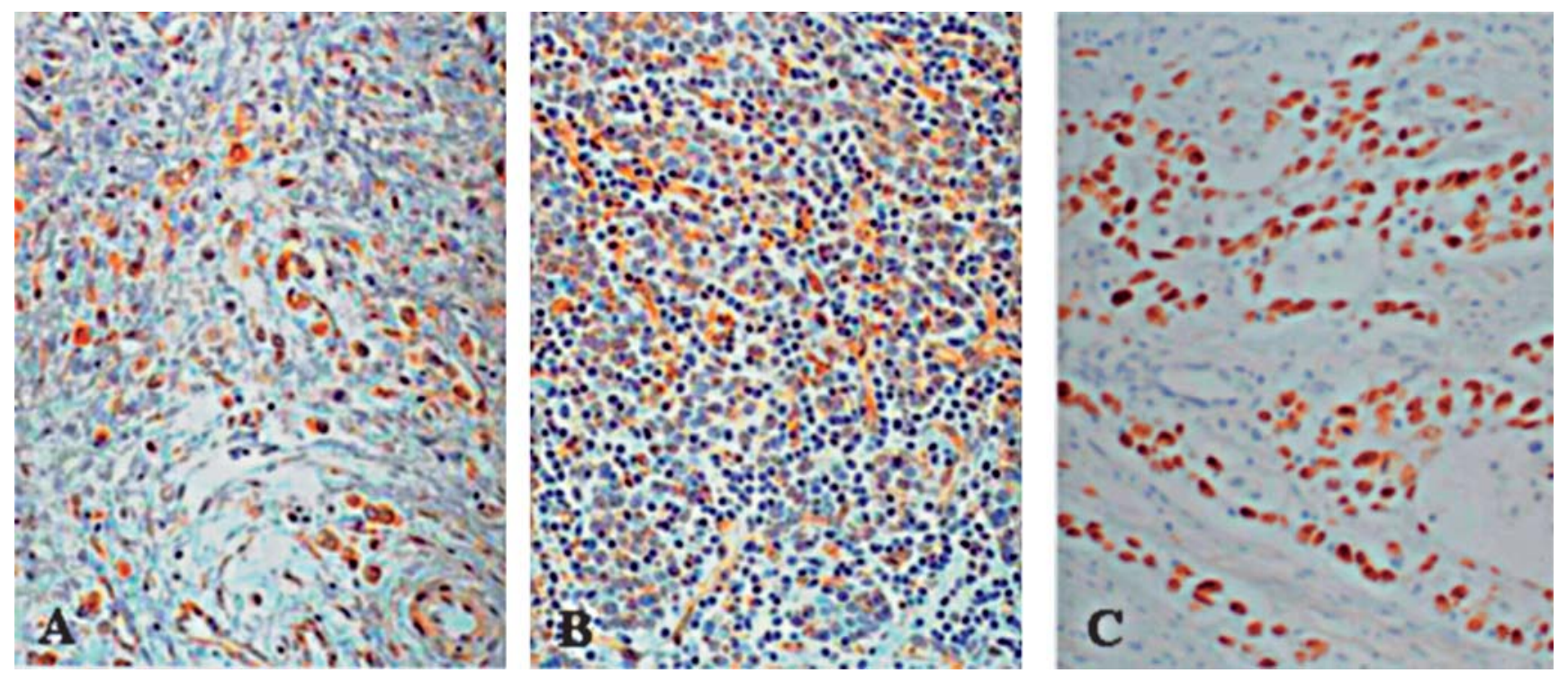

Figure 2. Strong VEGF expression in cancer cells and endothelial cells (A). Moderate eNOS expression in cancer cells and strong expression in endothelial cells (B). Strong p53 expression in cancer cells (C).

Correlation of microvessel density with TP, VEGF, eNOS, and p53 expression in gastric cancer (Table I). The TP expression of cancer cells was strongly associated with microvessel density $(\mathrm{p}=0.030)$, but the stromal TP expression was not. Among other factors known as having angiogenic potential, VEGF expression showed strong correlation with the microvessel density of the tumor $(\mathrm{p}<0.001)$, but eNOS expression showed a marginally significant association $(\mathrm{p}=0.055)$. On the contrary, p53 expression was not associated with microvessel density.

Correlation of TP expression with regional lymph node metastasis. In the 16 cases of no regional lymph node metastasis, low TP expression of the cancer cells was noted in 9 cases (19.2\%) and high TP expression of the cancer cells in 7 cases (26.9\%). Low TP expression of the stroma was noted in 10 cases $(23.8 \%)$ and high TP expression of the stroma in 6 cases (19.4\%). In the 18 cases of 1-6 regional lymph node metastases, low TP expression of the cancer cells was noted in 12 cases $(25.5 \%)$ and high TP expression of the cancer cells in 6 cases (23.1\%). Low TP expression of the stroma was noted in 8 cases $(19.1 \%)$ and high TP expression of the stroma in 10 cases $(32.2 \%)$. In the 39 cases of more than 7 regional lymph node metastases, low TP expression of the cancer cells was noted in 26 cases (55.3\%) and high TP expression of the cancer cells in 13 cases $(50.0 \%)$. Low TP expression of the stroma was noted in 24 cases $(57.1 \%)$ and high TP expression of the stroma in 15 cases $(48.4 \%)$. No significant 
Table I. Correlation of microvessel density with TP, VEGF, eNOS, and p53 expression in gastric cancer.

\begin{tabular}{lrrrr}
\hline & & \multicolumn{3}{c}{ Microvessel density } \\
\cline { 3 - 5 } Variables & No. (\%) & Mean & SD & p-value \\
\hline TP cancer cells & & & & $\mathbf{0 . 0 2 9}$ \\
Low & $47(64.4)$ & 84.47 & 29.63 & \\
High & $26(35.6)$ & 101.23 & 32.57 & \\
TP stroma & & & & 0.937 \\
Low & $42(57.5)$ & 90.69 & 29.88 & \\
High & $31(42.5)$ & 90.10 & 34.15 & \\
VEGF & & & & $<\mathbf{0 . 0 0 1}$ \\
Low & & & & \\
High & $22(30.1)$ & 63.59 & 19.45 & \\
eNOS & $51(69.9)$ & 102.02 & 28.62 & \\
Low & & & & 0.055 \\
High & $47(64.4)$ & 85.19 & 30.08 & \\
p53 & $26(35.6)$ & 99.92 & 32.46 & \\
Negative & $31(42.5)$ & 91.45 & 33.30 & \\
Positive & $42(57.5)$ & 89.69 & 30.56 & \\
\hline
\end{tabular}

Student t-test.

correlation was detected between lymph node metastasis and tumoral or stromal TP expression or VEGF/TP coexpression.

\section{Discussion}

TP is a mitogenic and angiogenic factor present in platelets. TP levels are markedly increased in tumor tissue compared with normal tissue in a variety of tumor types (9-13). In this study, TP expression was not detected in most of the nontumoral glandular epithelial cells except for 5 cases. Although a few researchers did not find a significant association between TP expression and prognosis in gastric carcinomas (22), many other researchers reported that positive TP counts showed highly significant association with invasion, hematogenous metastasis, peritoneal metastasis and worse prognosis $(8,9,12)$. In studies aimed at analysing a potential correlation between TP and malignancy it was shown that high TP-expressing tumors had higher microvessel density than low TP-expressing tumors $(7,10)$. Angiogenesis is an essential process for the primary tumor to grow and invade into the adjacent normal structures. Several studies suggested that intratumoral angiogenesis was an important prognostic factor in both operable and advanced inoperable gastric cancers $(9,23,24)$. A direct association of VEGF and/or of TP with increased intratumoral angiogenesis and poor outcome is rather well-established in several studies on gastric cancer $(10,16-19)$. The microvessel density of the tumor showed strong correlation with VEGF expression $(\mathrm{p}=0.000)$ in the present study, which supported the previous reports $(10,17,19)$. Most of the previous reports regarding TP expression in gastric cancers were mainly focused on cancer cells (9-13). However, there are a few reports which analysed TP in cancer cells and stroma separately $(12,13)$ and TP expression in the advanced gastric carcinoma has been barely studied. We assessed the TP expression of cancer cells and stroma, separately. The stromal TP expression was not associated with the TP expression of the cancer cells. The TP expression of the cancer cells was strongly associated with microvessel density ( $\mathrm{p}=0.030)$, but the stromal TP expression was not. Our result was consistent with those of the previous studies $(5,9,25,26)$. Although some previous studies reported that TP expression in infiltrating cells closely correlated with tumor vascularity $(16,27)$, a recent study by Yasuno et al suggested that TP produced by tumor cells has a stimulatory effect on tumor angiogenesis, while that produced by stromal cells plays an entierly different role (26). Some previous studies reported that there was a correlation between TP expression in cancer cells and lymph node metastasis also $(12,28)$. However, in our study, no significant correlation was detected between lymph node metastasis and tumoral and stromal TP expression or VEGF and TP coexpression. The different result of this study may be attributed to the different fixative, immunohistochemical method and analyzing criteria of the immunohistochemical staining result.

Mutations of the p53 protein have also been suggested to contribute to the angiogenic process through suppression of a strong inhibitor of angiogenesis, namely thrombospondine-1 (20). However, our study failed to reveal an association between angiogenesis and p53 expression. Although the role of eNOS in angiogenesis and progression of gastric cancer remains unclear, several studies reported concentrated localization of eNOS in the endothelial cells of normal gastric mucosa and tumor tissue $(14,15)$. The eNOS was more abundant in T1 and T2 tumors than in T3 and T4 tumors (29) and a comparative study of angiogenesis in early and advanced gastric cancers revealed that T1 tumor has higher values of neovascularization than T2 and T3 tumors (30). Although eNOS expression showed a marginally significant association with microvessel density ( $\mathrm{p}=0.055)$ in the present study, this result together with the above facts suggest that eNOS may enhance tumor angiogenesis in gastric cancer.

In gastric cancer, the TP expression of cancer cells but not stromal cells showed a significant correlation with increased microvessel density. The TP expression of cancer cells may play an important role in tumor growth by microvessel formation.

\section{Acknowledgements}

This study was supported by Institute of Biomedical Science and Technology (IBST-2004-5) of Konkuk University, Korea.

\section{References}

1. Furukawa T, Yoshimura A, Sumizawa T, et al: Angiogenic factor. Nature 356: 668, 1992.

2. Miyazono K, Okabe T, Urabe A, Takaku F and Heldin $\mathrm{CH}$ : Purfication and properties of an endothelial cell growth factor from human platelets. J Biol Chem 262: 4098-4103, 1987.

3. Ishikawa F, Miyazono K, Hellman U, et al: Identification of angiogenic activity and the cloning and expression of plateletderived endothelial cell growth factor. Nature 338: 557-562, 1989. 
4. Miyadera K, Sumizawa T, Haraguchi M, et al: Role of thymidine phosphorylase activity in the angiogenic effect of platelet-derived endothelial growth factor/thymidine phosphorylase. Cancer Res 55: 1687-1690, 1995.

5. Osaki M, Sakatani T, Okamoto E, Goto E, Adachi H and Ito H: Thymidine phosphorylase expression results in a decrease in apoptosis and increase in intratumoral microvessel density in human gastric carcinomas. Virchows Arch 437: 31-36, 2000.

6. Imazano Y, Takebayashi Y, Nishiyama K, et al: Correlation between thymidine phosphorylase expression and prognosis in human renal cell carcinoma. J Clin Oncol 15: 2570-2578, 1997.

7. Takebayashi Y, Akiyama S, Akiba S, Yamada K, Miyadera K and Sumizawa T: Clinicopathologic and prognostic significance of an angiogenic factor, thymidine phosphorylase, in human colorectal carcinoma. J Natl Cancer Inst 88: 1110-1117, 1996.

8. Shimaoka S, Matsushita S, Nitanda T, et al: The role of thymidine phosphorylase expression in the invasiveness of gastric carcinoma. Cancer 88: 2220-2227, 2000.

9. Kimura H, Konishi K, Nukui T, et al: Prognostic significance of expression of thymidine phosphorylase and vascular endothelial cell growth factor in human gastric carcinoma. J Surg Oncol 76: 31-36, 2001.

10. Giatromanolaki A, Koukourakis MI, Stathopoulos GP, et al: Angiogenic interactions of vascular endothelial growth factor, of thymidine phosphorylase, and of p53 protein expression in locally advanced gastric cancer. Oncol Res 12: 33-41, 2000.

11. Noguchi T, Fujiwara S, Takeno S, et al: Clinical impact of thymidine phosphorylase expression in gastric cancer. Oncol Rep 10: 561-566, 2003.

12. Konno S, Takebayashi Y, Higashimoto M, et al: Thymidine phosphorylase expression in gastric carcinoma as a marker for metastasis. Anticancer Res 23: 5011-5014, 2003.

13. Hotta T, Taniguchi K, Kobayashi Y, et al: Increased expression of thymidine phosphorylase in tumor tissue in proportion to TPexpression in primary normal tissue. Oncol Rep 12: 539-541, 2004.

14. Koh EJ, Noh SH, Lee YD, et al: Differential expression of nitric oxide synthase in human stomach cancer. Cancer Lett 146: 173-180, 1999

15. Kwon KM, Chu YC and Hwang TS: Expression of nitric oxide synthase isotypes in advanced gastric carcinoma. Korean J Pathol 36: 374-381, 2002

16. Takahashi Y, Cleary KR, Mai M, Kitadai Y, Bucana CD and Ellis LM: Significance of vessel count and vascular endothelial growth factor and its receptor (KDR) in intestinal-type gastric cancer. Clin Cancer Res 2: 1679-1684, 1996.
17. Takahashi Y, Bucana CD, Akagi Y, et al: Significance of platelet-derived endothelial cell growth factor in the angiogenesis of human gastric cancer. Clin Cancer Res 4: 429-434, 1998.

18. Maeda K, Chung YS, Ogawa Y, et al: Prognostic value of vascular endothelial growth factor expression in gastric carcinoma. Cancer 77: 858-863, 1996.

19. Tomoda M, Maehara Y, Kakeji Y, Ohno S, Ichiyoshi Y and Sugimachi K: Intratumoral neovascularization and growth pattern in early gastric carcinoma. Cancer 85: 2340-2346, 1999.

20. Dameron KN, Volpert OV, Tainsky MA and Bouck N: Control of angiogenesis fibroblasts by p53 regulation of thrombospondin- 1 . Science 265: 1582-1584, 1994.

21. Omejc M, Juvan R, Jelenc F and Repse S: Lymph node metastases in gastric cancer: correlation between new and old UICC TNM classification. Int Surg 86: 14-19, 2001.

22. Maeda K, Kang SM, Ogawa M, et al: Combined analysis of vascular endothelial growth factor and platelet-derived endothelial cell growth factor expression in gastric carcinoma. Int J Cancer 74: 545-550, 1997.

23. Xiangming C, Hokita S, Natsugoe S, et al: Angiogenesis as an unfavorable factor related to lymph node metastasis in early gastric cancer. Ann Surg Oncol 5: 585-589, 1998.

24. Araya M, Terashima M, Takagane A, et al: Microvessel count predicts metastasis and prognosis in patients with gastric cancer. J Surg Oncol 65: 232-236, 1997.

25. Sakatani T, Okamoto E, Tsujitani S, Ikeguchi M, Kaibara N and Ito H: Expressions of thymidine phosphorylase (dThdPase) and vascular endothelial growth factor on angiogenesis in intestinaltype gastric carcinoma. Oncol Rep 7: 831-836, 2000.

26. Yasuno M, Mori T, Koike M, et al: Importance of thymidine phosphorylase expression in tumor stroma as a prognostic factor in patients with advanced colorectal carcinoma. Oncol Rep 13: 405-412, 2005.

27. Tsujitani S, Saito H, Maeta Y, et al: Neoangiogenesis in patients with gastric carcinoma in relation to the expression of vascular endothelial growth factor and thymidine phosphorylase. Anticancer Res 24: 1853-1859, 2004.

28. Koide N, Nashio A, Hiraguri M, et al: Differences and relationships of thymidine phosphorylase expression in tumorassociated macrophages and cancer cells in squamous cell carcinoma of the esophagus. Dis Esophagus 15: 67-73, 2002.

29. Doi C, Noguchi Y, Marat D, et al: Expression of nitric oxide synthase in gastric cancer. Cancer Lett 144: 161-167, 1999.

30. Vindigni C, Miracco C, Spina D, et al: Cell proliferation, cell death and angiogenesis in early and advanced gastric cancer of intestinal type. Int J Cancer 74: 637-641, 1997. 